\title{
Analysis of Plates under Point Load Using Zener Material Model
}

\author{
Fethi Kadıŏlu*, Gülçin Tekin \\ Istanbul Technical University, Department of Civil Engineering, Istanbul, Turkey. \\ * Corresponding author. Tel.: +902122853706; email: fkadioglu@itu.edu.tr \\ Manuscript submitted June 30, 2017; accepted August 23, 2017. \\ doi:10.17706/ijcee.2017.9.2.484-491
}

\begin{abstract}
In this study, the mixed finite element method in the Laplace-Carson space is developed for viscoelastic plates under point load using Zener material model, utilizing the functional through a systematic procedure based on the Gâteaux Differential. The functional has four independent variables; deflection, two bending moments and one twisting moment in addition to the geometric and dynamic boundary conditions in Laplace-Carson space. The results obtained in the Laplace-Carson domain are converted to real time domain by inverse Laplace transform via Dubner \& Abate's and Durbin's algorithms. The performance of the developed solution technique is tested through various quasi-static problems.
\end{abstract}

Key words: Classical plate theory, Gâteaux differential, viscoelasticity, Zener material model.

\section{Introduction}

As a structural member, plates are extensively used in all fields of engineering. Therefore, many researchers have carried out extensive studies in the area of plate problem analysis. Based on the changes in structural materials, different plate theories are developed. While the theory of classical or Kirchhoff's plate theory omits the effect of the transverse shear deformation on the deflection of plate, Reissner-Mindlin plate theory considers the influence of it.

Hence there are many investigations about the behavior of the plate elements in the literature, they are restricted to the simple elastic constitutive relations. Due to the fact that real structural materials show time-dependent behavior and their response depends on the excitation history, viscoelastic constitutive relations should be considered in order to analyze the behavior of plate elements in correctly.

Philosophy and basic concepts of mechanical behavior of viscoelastic materials are explained in the literature [1], [2]. In structural analysis of time-dependent materials, linear viscoelasticity has been used for a long time. There are basically three approaches that can be used in linear viscoelastic analysis: Laplace transformation, Fourier transformation and Direct Time Integration method. [1] applied Laplace transform to viscoelastic beams. [2] presented the application of the Fourier transform to viscoelastic beams. [3] analyzed and compared the Time Integration methods in linear viscoelasticity.

For the problems that have complex geometry, loading conditions and material properties, closed-form solutions are often not possible. Therefore, numerical solution techniques should be employed. The application of the finite element method to the solution of viscoelastic problems has been presented by a number of authors [4]-[10].

When compared to conventional finite element methods, mixed finite element methods are more 
powerful and they provide solutions that are free from shear locking that is encountered as thickness of the plate element decreases. In the mixed finite element formation, Hellinger-Reissner and Hu-Washizu principles in addition to Gâteaux differential approach are widely used ones. The Gâteaux differential method has important advantages over the Hellinger-Reissner and Hu-Washizu principles. Comparison of these principles is widely discussed by [11], [12].

In this study, an analysis of viscoelastic plates by employing the Gâteaux differential approach is given. This approach has already been applied by the Aköz and his co-workers for the analysis of viscoelastic Timoshenko beams [13], viscoelastic parabolic beams [14], viscoelastic circular beams [15], [16] and viscoelastic Kirchhoff plates [17].

In this study, classical thin plates subjected to point load, made of the Zener viscoelastic material model are analyzed. The Maxwell and Kelvin models are the simplest viscoelastic models. More realistic material responses can be modelled using more elements. In contrast to the other well-known simpler Maxwell and Kelvin-Voigt models, Zener solid model is slightly more complex, involving elements (springs and damper) both in series and in parallel. In order to remove time derivatives, the method of Laplace-Carson transform is utilized. For numerical transformation of the solutions obtained in the Laplace-Carson domain to the real time domain, Dubner \& Abate's [18] and Durbin's [19] inverse Laplace transform methods are used. Numerical results for quasi-static response of viscoelastic thin plates are presented in representative problems.

\section{Method}

In the isotropic plate problems, the constitutive equation of viscoelastic materials has two different operators for dilatation and distortion. The use of two operators causes difficulties in solving problems. To overcome these difficulties, two simplifying assumptions are accepted in the literature. According to the first assumption, the dilatation is elastic and the distortion is viscoelastic. According to the second one, the distortion and dilatation parameters are proportional. Second assumption is equivalent to assuming that the Poisson ratio is constant.

Consider a plate as shown in Fig. 1, having a rectangular cross-section of thickness $h$. A Cartesian coordinate system $(x, y$, and $z)$ is defined at the central axis of the plate. Positive directions of the stress resultants are shown in Fig. 1.

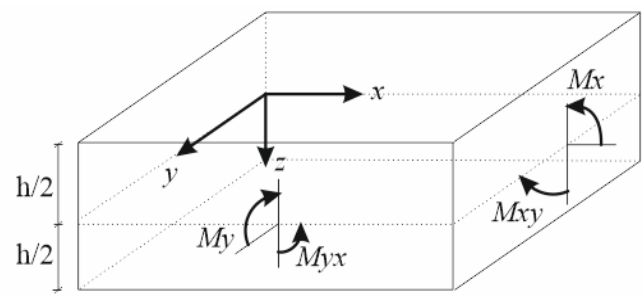

Fig. 1. Positive directions of stress resultants

In this study, assuming second assumption; the governing equations of viscoelastic Kirchhoff plates were derived in the Laplace-Carson domain by [17] as follows:

$$
\begin{aligned}
& \frac{\partial^{2} \bar{M}_{x}}{\partial x^{2}}+2 \frac{\partial^{2} \bar{M}_{x y}}{\partial x \partial y}+\frac{\partial^{2} \bar{M}_{y}}{\partial y^{2}}=0 \\
& \bar{M}_{x}=-\frac{h^{3}}{12}\left[\bar{E}_{1}^{*} \frac{\partial^{2} \bar{w}}{\partial x^{2}}+\bar{E}_{2}^{*} \frac{\partial^{2} \bar{w}}{\partial y^{2}}\right] \\
& \bar{M}_{y}=-\frac{h^{3}}{12}\left[\bar{E}_{1}^{*} \frac{\partial^{2} \bar{w}}{\partial y^{2}}+\bar{E}_{2}^{*} \frac{\partial^{2} \bar{w}}{\partial x^{2}}\right] \\
& \bar{M}_{x y}=-\frac{h^{3}}{12}\left[\bar{E}_{1}^{*}-\bar{E}_{2}^{*}\right] \frac{\partial^{2} \bar{w}}{\partial x \partial y} .
\end{aligned}
$$


The terms defined by the over bar notation are the transformed terms in the Laplace-Carson domain. The Laplace-Carson transform of a real function is defined as s-multiplied Laplace transform. Here, $M_{x}, M y, M x y$ are internal moments, $\mathrm{w}$ is the vertical displacement function of the plate' middle surface. To relate the stress-strain $(\sigma-\varepsilon)$ relations of viscoelastic plates' material, two operators $E_{1}^{*}$ and $E_{2}^{*}$ are defined. These operators are in the hereditary integral form. Explicit forms of these operators can be obtained from [17].

Field equations given in (1) can be written in the operator form as:

$$
\bar{Q}=\bar{L} \bar{y}-\bar{f} .
$$

where $\bar{Q}$ is the potential operator in the Laplace-Carson domain if the equality:

$$
<d \bar{Q}\left(\bar{y}, \bar{y}^{\prime}\right), \bar{y}^{*}>=<d \bar{Q}\left(\bar{y}, \bar{y}^{*}\right), \bar{y}^{\prime}>\text {. }
$$

is satisfied [20]. Gâteaux derivative of an operator is defined as:

$$
d \bar{Q}\left(\bar{y}, \bar{y}^{\prime}\right)=\left.\frac{\partial \bar{Q}\left(\bar{y}+\tau \bar{y}^{\prime}\right)}{\partial \tau}\right|_{\tau=0}
$$

where $\tau$ is a scalar. If the operator is potential then the functional corresponding to the field equations is obtained as:

$$
I(\bar{y})=\int_{0}^{1}[\bar{Q}(s \bar{y}), \bar{y}] d s .
$$

where s is a scalar quantity. Using (5), the functional corresponding to the operator in the Laplace-Carson domain becomes:

$$
\begin{aligned}
& I(\bar{y})=\left[\bar{w}_{, x}, \bar{M}_{x_{x}}\right]+\left[\bar{w}_{, y}, \bar{M}_{y_{y}}\right]+\left[\bar{w}_{, x}, \bar{M}_{x y, y}\right]+\left[\bar{w}_{, y}, \bar{M}_{x y_{x}}\right]-\frac{1}{2 \bar{D}\left(1-v^{2}\right)}\left\{\left[\bar{M}_{x}, \bar{M}_{x}\right]+\left[\bar{M}_{y}, \bar{M}_{y}\right]\right\} \\
& +\frac{v}{\bar{D}\left(1-v^{2}\right)}\left[\bar{M}_{x}, \bar{M}_{y}\right]-\frac{1}{\bar{D}(1-v)}\left[\bar{M}_{x y}, \bar{M}_{x y}\right]-[\hat{\bar{T}}, \bar{w}]_{\sigma}-\left[(\bar{M}-\hat{\bar{M}}), \bar{w}^{\prime}\right]_{\sigma}-\left[\left[\hat{\bar{w}}^{\prime}, \bar{M}\right]_{\varepsilon}-[(\bar{w}-\hat{\bar{w}}), \bar{T}]_{\varepsilon} .\right.
\end{aligned}
$$

Here, the square brackets represent the inner product and the parentheses with the subscripts $\sigma$ and $\varepsilon$ represents the dynamic and geometric boundary conditions of the plates, respectively. The quantities with hat are known values on the boundaries.

If a four-node rectangular master element as shown in Fig. 2 with interpolation functions given as below:

$$
\begin{aligned}
& N_{1}=(1-\xi)(1-\eta) \\
& N_{2}=\xi(1-\eta) \\
& N_{3}=(1-\xi) \eta \\
& N_{4}=\xi \eta .
\end{aligned}
$$

is used and all unknown quantities are expressed in terms of interpolation functions and then inserted into constructed functional in (6), the element matrix is obtained in the Laplace-Carson domain after extremization of the functional with respect to sixteen nodal variables. Here, the $(\xi, \eta)$ coordinates are mere translation of $(x, y)$. 


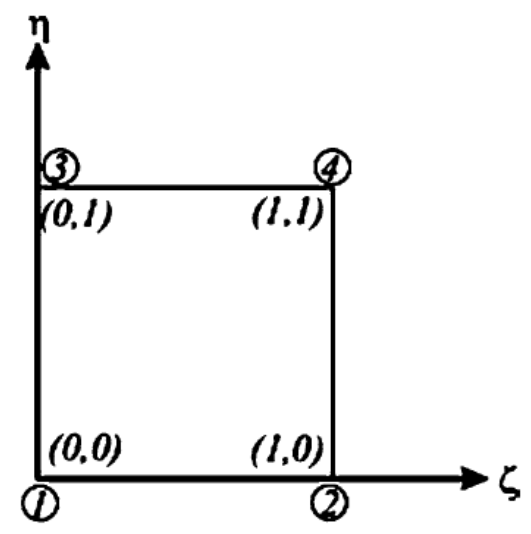

Fig. 2. Rectangular master element.

\section{Numerical Examples and Discussion}

Computations have been made for calculating the time-dependent displacement results of a viscoelastic rectangular plate. In the solution of viscoelastic thin plates, the Zener model is considered. The Zener model is represented by a spring-dashpot elements as illustrated in Fig. 3.

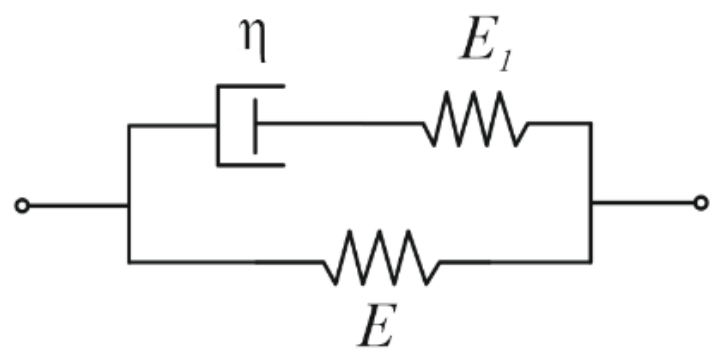

Fig. 3. Mechanical analog for the Zener model.

In this three-parameter model, a spring element is added in parallel to the Maxwell element. In calculations, the following material properties are used.

- $E=E_{1}=3 \times 10^{7} \mathrm{kPa}, \eta=3 \times 10^{7} \mathrm{kPa}$, Poisson's ratio $v=0.3$

For each problem, point load $\left(\mathrm{P}_{0}\right)$ is applied at the central point of the plate and a quarter of a simply supported plate where the length and width values are assumed to be $4 \mathrm{~m}$ is analyzed due to the symmetry. In all cases, the quarter domain has been divided into $16(4 \times 4)$ elements with equal sizes.

\subsection{Example 1}

In this example, the performance of the developed solution technique is tested. The present results are compared with those of [17]. This example has been treated by [17] for Kelvin-Voight model. The time-dependent displacement values at the plate center for a point load $\mathrm{P}_{0}=100 \mathrm{kN}$ are illustrated in Fig. 4 by employing Dubner \& Abate's inverse Laplace transform technique for a $T=5, N=100$ and $T=20 \mathrm{~s}$.

One can see that, the calculated results of the viscoelastic plate modeled with the Zener model are coincide with those given in [17]. In order to make comparison between the results of Kelvin-Voight model and Zener model, the modulus of elasticity of the spring which is in series arrangement to dashpot in the Zener model is changed. 


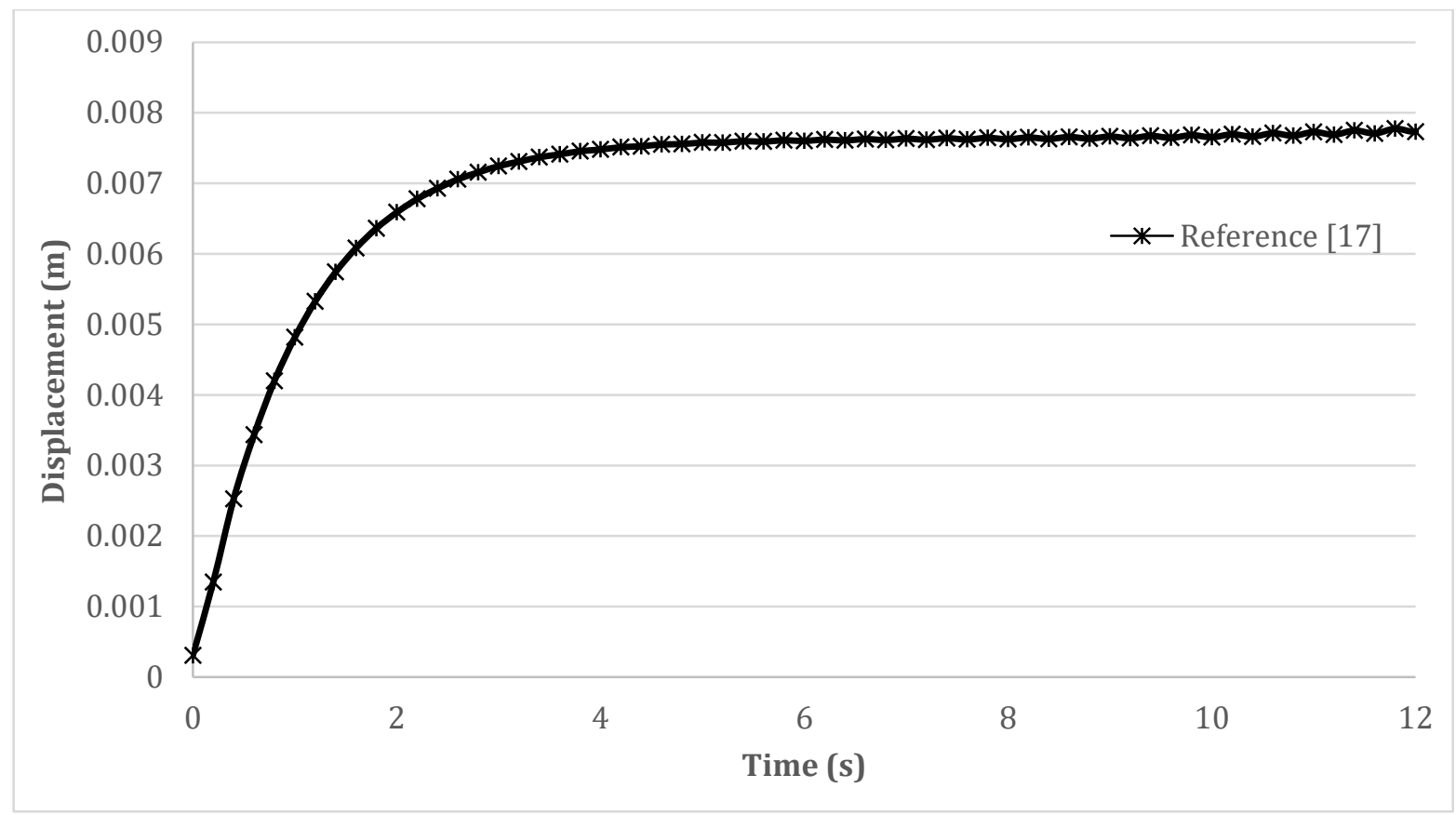

Fig. 4. Displacement results of viscoelastic plates under point load.

The Zener model is referred to as solid since it reacts instantaneously as elastic materials and recovers completely upon unloading. The displacement of Zener model approaches a finite value as $t \longrightarrow \infty$. Kelvin-Voight model is also characterizes a viscoelastic solid. The displacement of the Kelvin-Voight model approaches a finite value as $t \longrightarrow \infty$ whereas it does not have an elastic response to the suddenly applied load. The difference between these two viscoelastic solid models are illustrated in Fig. 5.

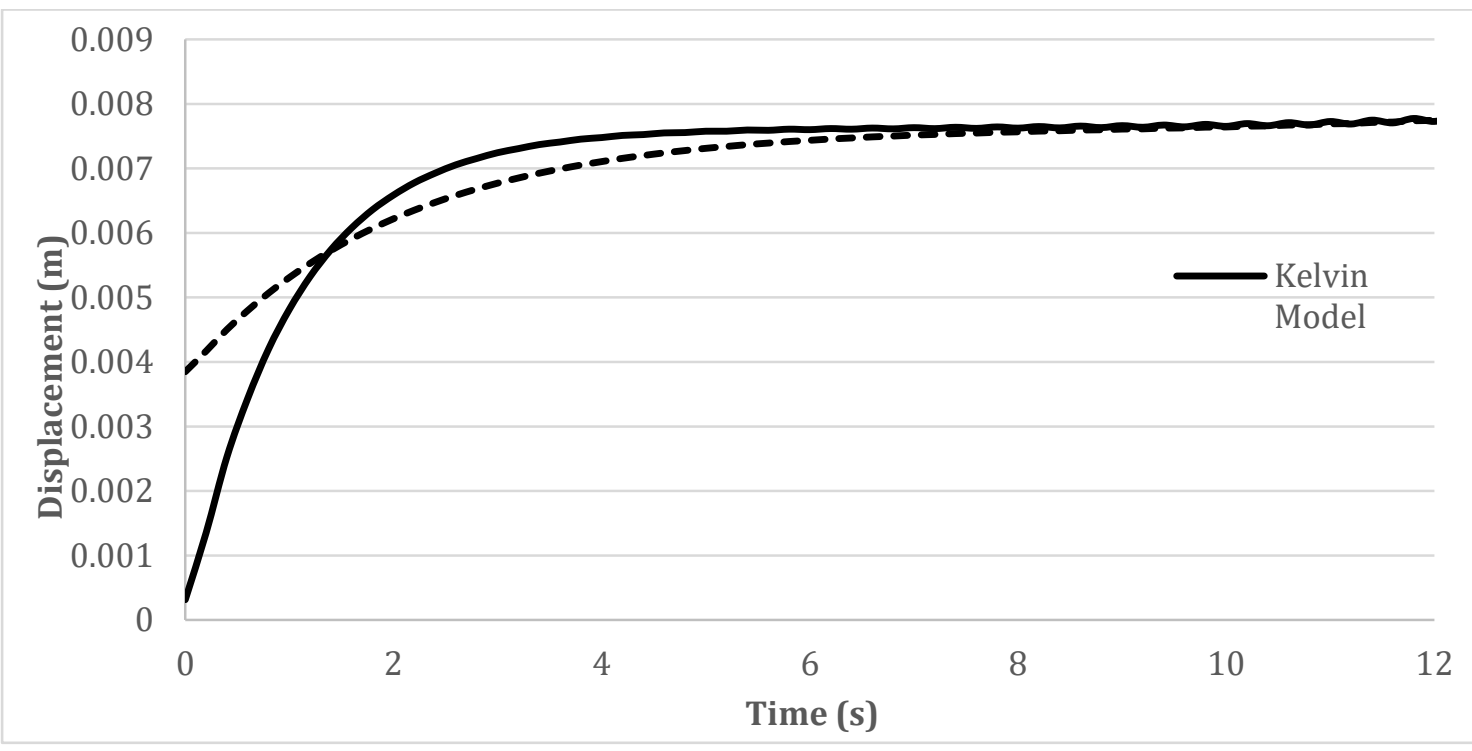

Fig. 5. Comparison of displacement results of Kelvin-Voight model and Zener model.

\subsection{Example 2}

A viscoelastic plate subjected to the point load is considered for different thickness values. The point load of interest has a form of a rectangular pulse; a constant magnitude $P_{0}=100 \mathrm{kN}$ with a duration of $10 \mathrm{~s}$. This example shows the effect of the thickness variation on the behavior of the viscoelastic plates. For the 
thickness of the plate $h$, three different values: $h=0.05 \mathrm{~m}, h=0.075 \mathrm{~m}$ and $h=0.1 \mathrm{~m}$ are assumed. In Fig. 6 time variation of displacement values at the center of the plate are shown for three values of $h$. The results are computed by employing Durbin's inverse Laplace transform technique for a $T=5, N=200$ and $T=40 \mathrm{~s}$.

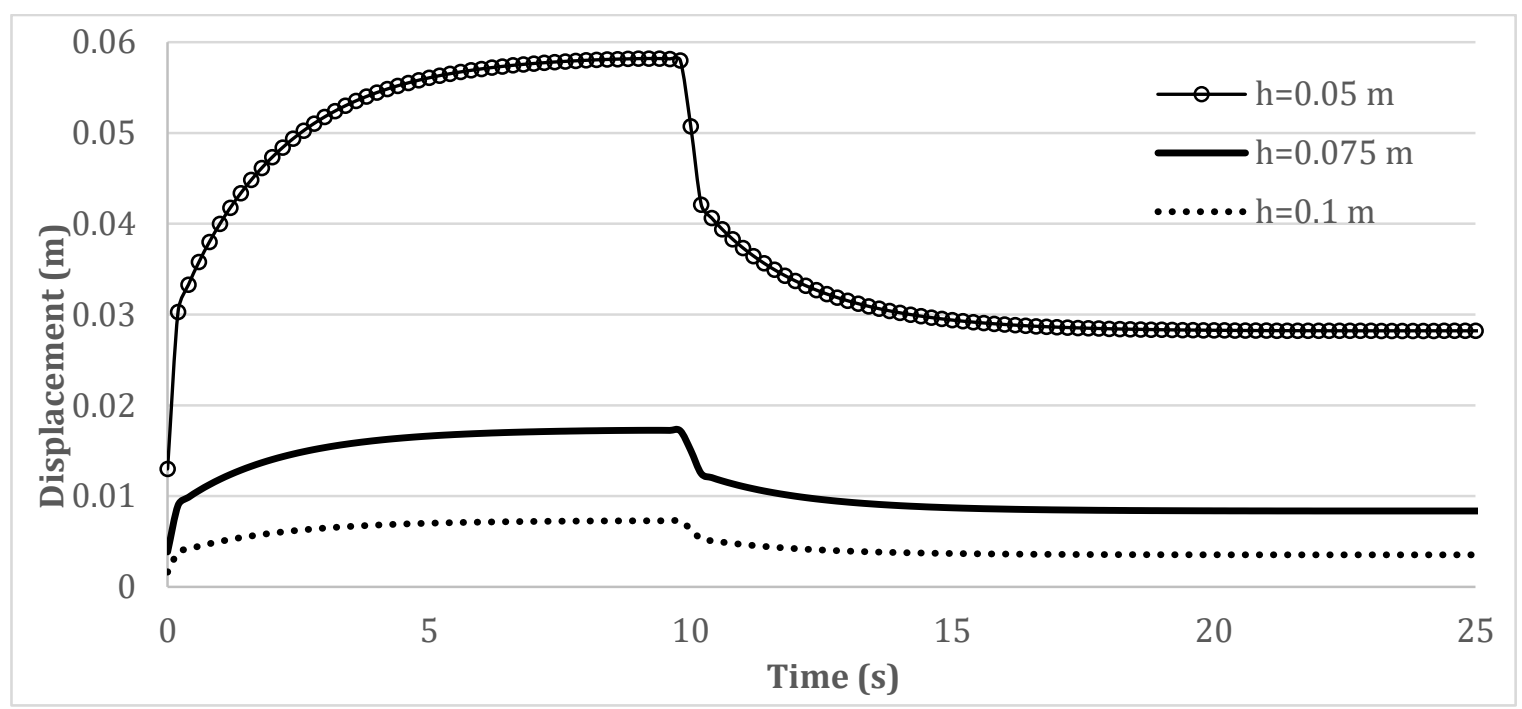

Fig. 6. Effect of different values of plate thickness under point load which has a form of a rectangular pulse.

As expected, decreasing the thickness of the plate causes increase in the time-dependent displacement values. Moreover, decreasing the thickness of the plate element does not cause shear locking.

\subsection{Example 3}

In this example, a viscoelastic plate loaded by a point load $P_{0}=100 \mathrm{kN}$ is considered. The effect of increasing the viscosity coefficient on the response is shown in Fig. 7. The results are computed by employing Dubner \& Abate's inverse Laplace transform technique for a $T=5, N=200$ and $T=40 \mathrm{~s}$. When the viscosity coefficient decreases, the time-dependent displacement behavior of the plate approaches the elastic behavior as expected.

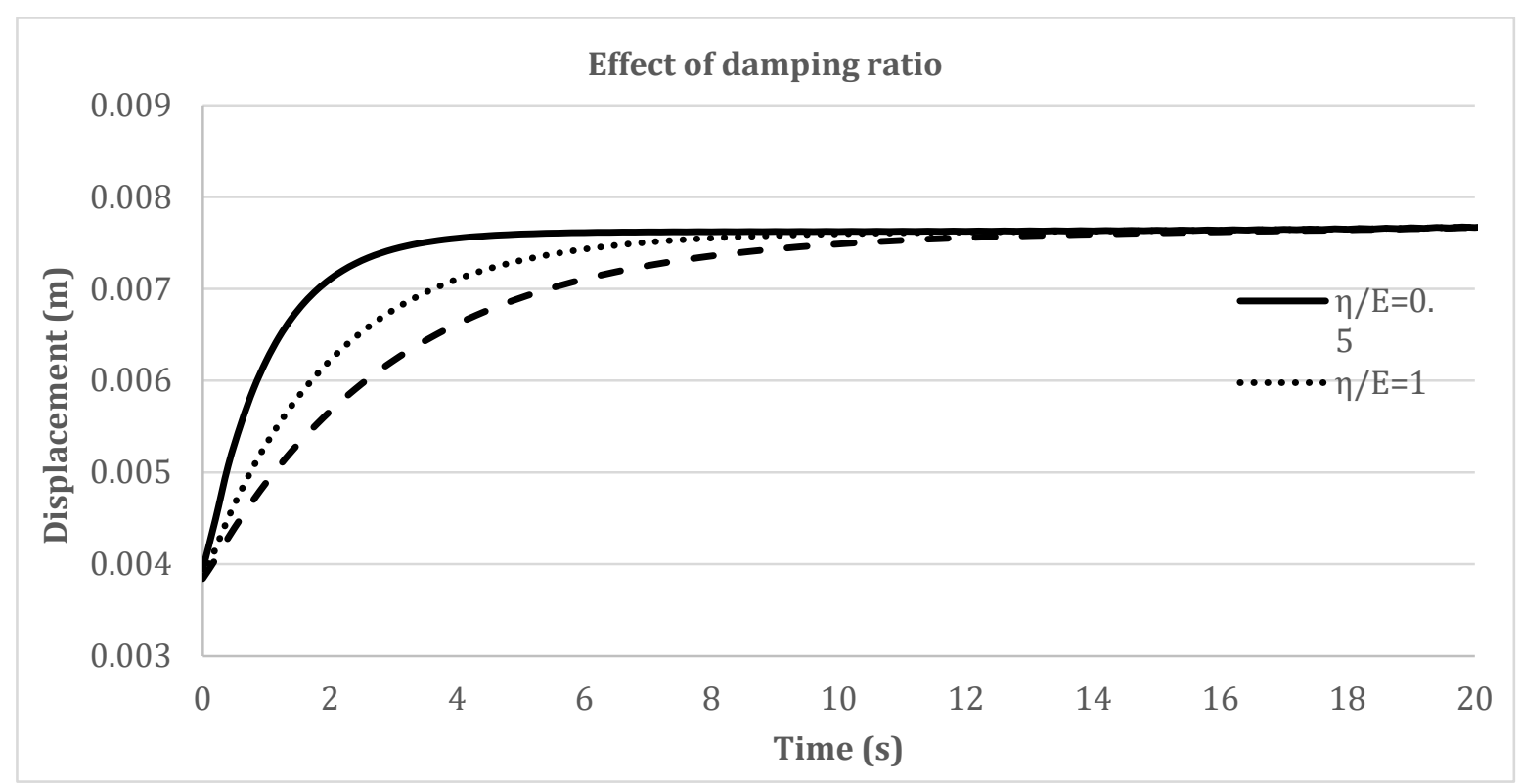

Fig. 7. Effect of the different $\eta / E$ ratios under point load. 


\section{Conclusion}

In this paper, a functional with the necessary boundary condition terms for the analysis of viscoelastic classical thin plates subjected to point loads is constructed using a systematic procedure based on the Gâteaux differential approach. Mixed-type finite element composed of four nodes, each with four degrees of freedom, is formulated for this analysis. By the quasi static analysis of square plates loaded by a point load (Example 1), the performance of the proposed solution technique is tested by comparing the results available in literature and the calculated results and a very good accuracy is obtained. In addition by Example 1, time-dependent displacement results of two different mechanical viscoelastic models are presented. Using plate elements with different thickness values, it is showed that the presented mixed finite element formulation avoids shear locking (Example 2). Also by Example 3, the influence of the damping ratio ( $\eta$ / E ratio) on the quasi static response is investigated, since this ratio is one of the most important factors affecting the viscoelastic behaviors. It is thought that the results of this paper may serve as a benchmark and may be useful for scientists and researchers in assisting them to evaluate the accuracy of their further investigation results for comparison purposes. The same approach can be applied for higher order plate theories as well as shell theories loaded by a point load. Following the described methodology, some of these problems are under study.

\section{Acknowledgment}

The authors thank Scientific and Technological Research Council of Turkey that supported the present work under the grant number 213M332.

\section{References}

[1] Flügge, W. (1975). Viscoelasticity (2nd ed.). Berlin: Springer.

[2] Christensen, R. M. (1982). Theory of Viscoelasticity (2nd ed.). New York: Academic Press.

[3] Sorvari, J., \& Hämäläinen, J. (2010). Time integration in linear viscoelasticity - A comparative study. Mechanics of Time Dependent Materials, 14, 307-328.

[4] White, J. L. (1986). Finite elements in linear viscoelastic analysis. Proceedings of the 2nd Conference on Matrix Method in Structural Mechanics (pp. 489 - 516). AFFDL-TR-68-150.

[5] Chen, T. M. (1995). The hybrid Laplace transform / finite element method applied to the quasi-static and dynamic analysis of viscoelastic Timoshenko beams. International Journal for Numerical Methods in Engineering, 38, 509-522.

[6] Wang, Y. Z., \& Tsai, T. J. (1988). Static and dynamic analysis of a viscoelastic plate by the finite element method. Applied Acoustics, 25, 77-94.

[7] Yi, S., Hilton, H. H. (1994). Dynamic finite element analysis of viscoelastic composite plates. International Journal for Numerical Methods in Engineering, 37, 4081-96.

[8] Nguyen, S. N., Lee, J., \& Cho, M. (2016). A triangular finite element using Laplace transform for viscoelastic laminated composite plates based on efficient higher-order zigzag theory. Composite Structures, 155, 223-244.

[9] Temel, B., \& Sahan, M. F. (2013). An alternative solution method for the damped response of laminated Mindlin plates. Composites Part B-Engineering, 47, 107-117.

[10] Attia, M. A., El-Shafei, A. G., \& Mahmoud, F. F. (2014). Nonlinear analysis of frictional thermos-viscoelastic contact problems using FEM. International Journal of Applied Mechanics, 6, 1450028.

[11] Aköz, A.Y., \& Özütok, A. (2000). A functional for shells of arbitrary geometry and a mixed finite element method for parabolic and circular cylindrical shells. International Journal for Numerical Methods in 
Engineering, 47, 1933-1981.

[12] Ateş, N., Tekin, G., \& Kadığlu, F. (2016). Alternative solution for cross-ply laminated composite thick plates. Gradevinar, 68(6), 451-460.

[13] Aköz, A. Y., \& Kadığlu, F. (1999). The mixed finite element method for the quasi-static and dynamic analysis of viscoelastic Timoshenko beams. International Journal for Numerical Methods in Engineering, 44, 1909-1932.

[14] Kadığlu, F., \& Aköz, A. Y. (2000). The quasi-static and dynamic responses of viscoelastic parabolic beams. Proceedings of the 11th National Applied Mechanics Meeting (in Turkish), Bolu-Turkey.

[15] Kadığlu, F., \& Aköz, A. Y. (1999). The mixed finite element method for the dynamic analysis of visco-elastic circular beams. Proceedings of the 4th International Conference on Vibration Problems, Jadavpur University.

[16] Kadığlu, F., \& Aköz, A. Y. (2003). The mixed finite element for the quasi-static and dynamic analysis of viscoelastic circular beams. International Journal of Structural Engineering and Mechanics, 15, 735-752.

[17] Aköz, A. Y., Kadıoğlu, F., \& Tekin, G. (2015). Quasi-static and dynamic analysis of viscoelastic plates. Mechanics of Time-Dependent Materials, 19 (4), 483-503.

[18] Dubner, H., \& Abate, J. (1968). Numerical inversion of Laplace transforms by relating them to the finite Fourier cosine transform. Journal of the Association for Computing Machinery, 15, 115-123.

[19] Durbin, F. (1974). Numerical inversion of Laplace transforms: An efficient improvement to Dubner and Abate's method. The Computer Journal, 17, 371-376.

[20] Oden, J. T., \& Reddy, J. N. (1976). Variational Methods in Theoretical Mechanics. Berlin, Springer.

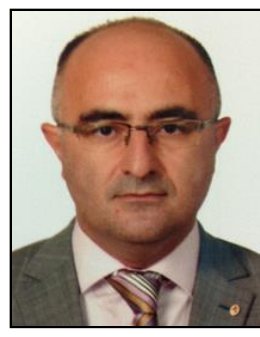

Fethi Kadıoğlu received his BSc, MSc and Ph.D from Istanbul Technical University, Istanbul, Turkey. He is currently working as an associate professor in the Department of Civil Engineering at Istanbul Technical University.

He published over 35 papers in international journals and conferences, 5 books and book chapters in the fields of Structural Mechanics.

Dr. Kadığlu's current research interests include: composite structures, stability of structures and vibration, elastic, viscoelastic analysis, structural analysis and finite element methods.

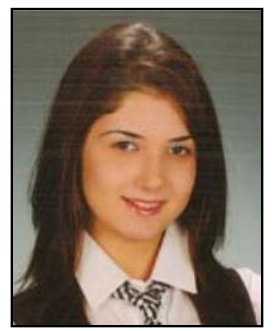

Gülçin Tekin received her BSc degree in civil engineering from Yıldız Technical University, Istanbul, Turkey. She completed MSc degree at the Boğaziçi University, Istanbul, Turkey. She is currently a Ph.D candidate in the Department of Civil Engineering at the Istanbul Technical University, Istanbul, Turkey.

Presently she is working as a research assistant at Istanbul Technical University. Her areas of interest are structural analysis, composite structures, elastic and viscoelastic analysis and finite element methods. 